\title{
Effectiveness of Brainwave Synchronization in Alpha, Beta, and Theta Bands by Binaural Beats on Visuospatial Working Memory
}

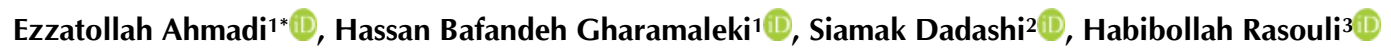 \\ 1 Associate Professor, Department of Psychology, Azarbaijan Shahid Madani University, Tabriz, Iran \\ 2 PhD student of Cognitive Neuroscience, Division of Cognitive Neuroscience, University of Tabriz \\ ${ }^{3}$ MSc in Cognitive Science, Department of Psychology, Azarbaijan Shahid Madani University, Tabriz, Iran
}

\author{
*Corresponding author: \\ Ezzatollah Ahmadi, Department of \\ Psychology, Azarbaijan Shahid \\ Madani University, Tabriz, Iran \\ Tel: 09141046002 \\ Email: amiraliahmadi91@yahoo.com.
}

Received: 07 Sep. 2020

Accepted: 05 Feb. 2021

ePublished: 01 Nov. 2021

\begin{abstract}
Background and Objective: This study aimed to determine the effect of synchronization of brain waves in alpha, beta, and theta bands by the Binaural beats on visuospatial working memory.

Materials and Methods: The present quasi-experimental study was conducted based on a pretestposttest control group design. In this regard, 60 students were selected by the available sampling method and randomly divided into three experimental groups and one control group ( $n=15$ each). All subjects were assessed in the pre-test and post-test stages by the Corsi blocks tapping test. Participants in the experimental groups received $15,9,5$, and $6 \mathrm{~Hz}$ binaural beats for $12 \mathrm{~min}$. The collected data were analyzed using a one-way analysis of covariance.

Results: The findings showed that $15 \mathrm{~Hz}$ binaural beats (beta bands) significantly improved the subject's visuospatial working memory $(\mathrm{P}<0.05)$.

Conclusions: Given that working memory is the basis of numerous cognitive functions, using $15 \mathrm{~Hz}$ binaural beats could improve the visuospatial working memory in these people.

Keywords: Brain waves, Cognitive functions, Working memory
\end{abstract}

\section{Background}

The brain is a very complex network of nonlinear systems with internal dynamic states that are not easily measurable. The networking nature of the brain is very much related to different functional tasks, such as memory, which is built up in scattered areas of the brain [1].

There are several classifications for memory, including sensory, short-term, and long-term memory. In recent decades, the term of working memory has replaced short-term memory. Working memory includes the ability to store and manipulate information for a short term and can be divided into subcategories of primary encoding, information, and storage and retrieval of materials [2]. People have limited capacity for information that can be stored in the working memory in a given time. Neuroanatomically, the working memory network is distributed over a large part of the brain. In particular, the task of working memory involved in visuospatial information activates the prefrontal cortex [3]. In addition, prefrontal and parietal neuronal accumulation are activated simultaneously during the maintenance of working memory [4]. Increased difficulty in a working memory task is associated with increased communication between the frontal and parietal areas [5].

Cortical activity can be recorded non-invasively on the scalp using electroencephalography (EEG). Since the EEG is nonlinear and unstable, phase synchronization has been well studied to determine the cortical networks. Phase synchronization is a statistical method of measuring the interdependence of two oscillators and it is used in the field of nonlinear dynamics and irregular systems [6]. Synchronization of the short-term or localized phase in the brain can be interpreted as the cause of the regional perception link [7]. Synchronization of the long-term phase between areas includes motor planning [8], excitement [9], and memory [10].

The results of previous research have shown that an increase in the synchronization of the electrocortical stage across the cortex facilitates neuronal connections, enhances neuronal morphology, and supports working memory [10]. The synchronized firing of pre-synapse neurons increases the rate of post-synapse neuron firing [11]. Synchronized gamma oscillations are limited to the local neuronal region, while theta synchronization is more effective 
over long distances (e.g., different areas of the brain). Successful data encryption during a work memory task requires increasing step synchronization [12]. Phase synchronization in the theta band is maintained between the prefrontal and parietal areas during a working memory task over encryption, maintenance, and retrieval [13]. Beta and gamma synchronizations, due to excitation, increase the coherence between the forehead and parietal areas while maintaining working memory [14]. Synchronization can be non-invasive by providing a special sound stimulus called binaural beats. Two-way beats require two different soundtracks to be heard [15]. This method causes a beat of the two-third phones, the frequency of which is equal to the difference between the two tones provided, which is produced in the Inferior Colliculus (IC) located in the auditory canal [16]. General phase difference from IC to auditory cortex with periodic nerve firing is maintained at binaural beats frequencies [17]. The highest synchronization rate occurs due to the binaural beats in the auditory cortex on the beta band at $16 \mathrm{~Hz}$ [18].

Based on the findings of previous studies, binaural beats affect the cortical responses throughout the frequency bands. In the gamma band, the largest EEG steady-state responses occur at a rate of 40 $\mathrm{Hz}$ and it mainly activates the frontal and parietal lobes. In addition, the stimulation of binaural beats in the beta band on $5.18 \mathrm{~Hz}$, increases EEG size by $21 \%$ [19]. The cortical areas are subjected to the binaural beats theta bands, including the parietal, frontal, and temporal regions [20].

The results of the research have shown that the binaural beats affect many aspects of cognition and mood, such as attention, memory, creativity, and alertness [21]. Although this method offers a non-invasive and comfortable stimulus, its effect on working memory has only been studied in a limited number of studies. For example, 22. Beauchene et al. [22] indicated that the binaural beats of $15 \mathrm{~Hz}$ could strengthen the accuracy and key connections in cortical networks in a task working memory. Klimesch et al. [23] showed that fluctuations in the alpha rhythm indirectly increased the performance of working memory capacity. So that they filter out inappropriate information and prevent the disruption of processes in the form of contradictory stimuli. On the other hand, Beauchene et al. [24] showed that the binaural beats on the beta band at $15 \mathrm{~Hz}$ during the execution of the working memory task activated significant frequency bands and created networks that were characterized by the transmission of more information. Carter and Russell [25] exposed 8-12-year-old boys with learning disabilities to $10 \mathrm{~Hz}$ and $18 \mathrm{~Hz}$ binaural beats stimulation sessions for 8 weeks and found an improvement in Raven's progressive matrices and in a subtest of auditory sequential memory. McMurray [26] evaluated the multiplication of Duchess 7 and $11 \mathrm{~Hz}$ on Alpha's brain wave, working memory, and the attention of healthy elderly people. Exposure to double beeping for 2 min causes a change in the electrical activity of the brain. In particular, changes in alpha wave activity occurred. In addition, there have been advances in the direct and indirect digit span tasks and in a part of the continuous performance test. Dai et al. [27] also showed that there was a higher functional integration in the theta band in the task of working memory than in the control group.

According to the above, the binaural beats can induce neuronal fluctuations and affect various cognitive functions, including working memory. On the other hand, research on the use of different frequencies to improve working memory shows conflicting results. Moreover, a more appropriate and specific band is not specified to enhance working memory.

\section{Objectives}

Therefore, this study aimed to investigate the differences between the effects of stimulating the alpha, beta, and theta bands by binaural beats on students' working memory?

\section{Materials and Methods}

The present quasi-experimental study was conducted based on a pretest-posttest control group design. The statistical population of the present study included all students aged 19 to 25 years at Azerbaijan Shahid Madani University, Tabriz, East Azerbaijan Province, Iran, studying for a bachelor's degree in the 2018-19 academic year. The samples $(\mathrm{n}=60)$ were selected using an available sampling method and then randomly assigned to three experimental groups and a control group. The exclusion criteria from the study were taking psychiatric medications and having a major depressive disorder, anxiety disorders, a history of brain trauma, a history of epilepsy or suffering from it, and heart disease. The collected data were analyzed in SPSS software (version 18) using a oneway analysis of covariance.

\section{Research tools}

Corsi block tapping test:

The Corsi block tapping test was developed and used in the early 1970s [28]. This test is designed based on the Digit Span test; however, this test requires the use of visuospatial working memory rather than the 
verbal form available in the Digit Span test. Functional magnetic resonance imaging studies on the tests that were being performed on the Corsi test have indicated that an increase in the number of sequences and stimuli of this test does not change the overall activity of your brain [29]. Consequently, while people may have trouble encoding, this increase in test levels has no relationship with general brain activation. In the general case, in the direct Span of Corsi test, we need the support of a visuospatial sketchpad and there is no need for a phonological ring, and when the sequence of recovery cases is more than three or four items, the central executive resources are used [30].

Within the process of the Corsi test, the subject sees nine blocks on the computer screen that in each attempt, several of these blocks are illuminated with a specific sequence. The subject's task is to remember the sequence of blocks being lit. Afterward, the subject repeats the sequence by clicking on the blocks. This test starts with two blocks and gradually increases the number of bright blocks in each attempt until it reaches 9 blocks. If the subject makes a mistake twice in a sequence, the test ends, and the longest sequence reminded by the subject is recorded. In general, the average reminder for normal people is five blocks [31]. The reliability of this test has been reported to be 0.73 [32].

\section{Binaural beats stimulation}

The Binaural beat frequency utilized in this study is equal to the difference between the frequencies used. In this regard, the following frequencies were produced by Audacity software (version 2.2) and Adobe Audition CC (version 2017) and high-quality wireless headphones: 1) for the right ear $=220 / 5 \mathrm{~Hz}$ and for the left ear $=230 \mathrm{~Hz}(9.5 \mathrm{~Hz}$ - alpha range $)$ according to the protocol used by Kraus and Probanova [33], 2) for the right ear $=240 \mathrm{~Hz}$ and for the left ear $=255 \mathrm{~Hz}(15 \mathrm{~Hz}$ - beta range $)$ according to the protocol used by Biochan et al. [22], and 3) for the left ear $=250 \mathrm{~Hz}$ and for the right ear $=256 \mathrm{~Hz}$ ( $6 \mathrm{~Hz}$ - theta range) according to the protocol used by Giracitiaacorn and Wangsawat [34].

Research method

The subjects were explained about the research procedure and provided their informed consent to perform brain wave synchronization. It was ensured that the subjects lacked a history of heart disease and epilepsy (to perform brain sound stimulation). Subsequently, the Corsi block tapping test, as a pretest of visuospatial working memory, was applied to subjects in all four groups. At the next stage, according to the protocol provided by Kraus and Probanova [33], the sound stimulation was presented to the subjects of the experimental groups for 12 min. The subjects of the control group were not stimulated. After that, the Corsi block tapping test was reperformed on the subjects of all three groups.

Results

The present study involved 60 students, and all subjects in four groups had the same education level and were undergraduate students. All participants in the pre-test and post-test stages were evaluated in terms of visuospatial working memory by the Corsi block tapping test. One-way analysis of covariance (ANCOVA) was used to perform statistical analysis. Before the ANCOVA test, its assumptions were examined. The results of the Kolmogorov-Smirnov test showed that the data had a normal distribution (sig=0.11, $z=1.19$ ). Based on the results of Levene's test, the assumption of homogeneity of variances was observed $(\operatorname{sig}=0.074, \mathrm{f}=0.001)$.

Table 1 presents the descriptive indicators of working memory in the studied groups in the pretest and post-test stages.

Descriptive findings indicated an increase in the working memory scores of the experimental group in the post-test stage.

To investigate the significance of the differences in group scores, a one-way covariance analysis test was employed, the results of which are summarized in Table 2.

As shown in Table 2, after deleting the pre-test effect, there was a significant difference in the posttest scores between the experimental group and the control group $(\mathrm{P}<0.05)$. Accordingly, synchronizing brainwaves improved visuospatial working memory and enhanced working memory capacity. Moreover, the square of Eta was obtained at 0.14 .

As the Bonferroni test shows, there was a significant difference between the brain wave synchronization groups in the beta band and the control group $(\mathrm{P}=0.30)$. However, there was no significant difference between alpha and beta, beta and theta, alpha and theta, alpha and control groups, and theta and beta $(\mathrm{P}<0.05)$.

Table 1. Mean and SD of visuospatial working memory per group

\begin{tabular}{|c|c|c|c|c|c|}
\hline \multirow{2}{*}{ Variable } & & \multicolumn{2}{|c|}{ Pretest } & \multicolumn{2}{|c|}{ Posttest } \\
\hline & & Mean & SD & Mean & SD \\
\hline Visuospatial working memory & Alpha stimulation & 5.50 & 0.75 & 5.83 & 0.77 \\
\hline Visuospatial working memory & Beta stimulation & 5.63 & 0.74 & 6.64 & 0.76 \\
\hline Visuospatial working memory & Theta stimulation & 5.43 & 0.75 & 6.03 & 1.12 \\
\hline Visuospatial working memory & Control & 5.60 & 0.80 & 5.66 & 0.89 \\
\hline
\end{tabular}


Table 2. Results of one-way analysis of covariance of the research variables

\begin{tabular}{|c|c|c|c|c|c|c|}
\hline Source & Mean Square & df & $\mathbf{F}$ & Sig. & Partial Eta Squared & Statistical Power \\
\hline Pre-visuospatial & 16.640 & 1 & 31.565 & 0.000 & 0.365 & \\
\hline Group & 1.663 & 3 & 3.154 & 0.032 & 0.147 & 0.701 \\
\hline Error & 0.527 & 55 & & & & \\
\hline
\end{tabular}

Table3. Bonferroni test for pairwise difference of mean spatial working memory scores between groups

\begin{tabular}{|c|c|c|c|c|}
\hline (I) Group & (J) Group & Mean Difference (I-J) & Std. Error & Sig. ${ }^{a}$ \\
\hline \multirow{3}{*}{ Alpha } & Beta & -0.538 & 0.266 & 0.286 \\
\hline & Theta & -0.248 & 0.265 & 1.000 \\
\hline & Control & 0.238 & 0.265 & 1.000 \\
\hline \multirow{3}{*}{ Beta } & Aplha & 0.538 & 0.266 & 0.286 \\
\hline & Theta & 0.291 & 0.266 & 1.000 \\
\hline & Control & $0.776^{*}$ & 0.265 & 0.030 \\
\hline \multirow{3}{*}{ Theta } & Aplha & 0.248 & 0.265 & 1.000 \\
\hline & Beta & -0.291 & 0.266 & 1.000 \\
\hline & Control & 0.485 & 0.266 & 0.440 \\
\hline \multirow{3}{*}{ Control } & Aplha & -0.238 & 0.265 & 1.000 \\
\hline & Beta & $-0.776^{*}$ & 0.265 & 0.030 \\
\hline & Theta & -0.485 & 0.266 & 0.440 \\
\hline
\end{tabular}

*Significant difference between groups $(\mathrm{P}<0.05)$

\section{Discussion}

This study aimed to compare the effect of brain wave synchronization on alpha, beta, and theta bands by Binaural beat on visuospatial working memory. The results of this study showed that the use of brain wave synchronization in beta bands could improve visuospatial working memory better than other alpha and theta bands. The findings of this study are consistent with those of studies conducted by Dadashi et al. [35], Beauchene et al. [22], Beauchene et al. [24], and Lane et al. [36]. Dadashi et al. [35] investigated the effect of synchronization of brain waves in beta band on people with borderline personality disorder symptoms and reported that $15 \mathrm{~Hz}$ Binaural beats increased the capacity of visuospatial working memory. Beauchene et al. [22] showed that synchronization of brain waves in the beta band $(15 \mathrm{~Hz})$ increased the accuracy of response in the visuospatial working memory task. Furthermore, Beauchene et al. [24] indicated that binaural beats in the beta band $(15 \mathrm{~Hz})$ significantly changed the relative accuracy of working memory. Lane et al. [36] also showed that listening to binaural beats on the beta band, during performing the task of working memory improved the tracking of the target. According to the present study, providing binaural beats on a beta band and a frequency of $15 \mathrm{~Hz}$ improved the working memory capacity of people with a borderline personality disorder. This increased performance in visuospatial working memory can be explained by the point that $15 \mathrm{~Hz}$ binaural beats generate high synchronization in the auditory cortex [18]. Additionally, according to the findings of Beauchene et al. [22], $15 \mathrm{~Hz}$ binaural beats make at least a partial change in the power of the network connection between the storage and retrieval sections of the working memory. Therefore, networks are better maintained throughout the task of working memory. It seems that the maintenance of working memory is guided by vibrating rings that cause stable nerve firing, and consequently, allows cognitive representations to be maintained in consciousness [37].

The results of a study have shown that beta activity increases during working memory retention [38]. As a result, synchronization of brain waves in the beta band can improve working memory. On the other hand, frontoparietal areas play an important role in visuospatial working memory. Based on the findings of another research, synchronization of brain waves in the beta band increases coherence between frontoparietal areas during working memory retention [14]. Therefore, it can be said that providing a frequency of $15 \mathrm{~Hz}$ in the beta band, improves working memory by increasing coherence between frontoparietal areas, which is in line with the results of the present study.

Given that the alpha wave does not lead to a significant change in visuospatial working memory, alpha waves can be said to act like sleep spindles and inhibit information from sensory systems at the thalamic and primary cortical levels [39]. The recording of single thalamocortical relay cells that are responsible for producing sleep spindles, an oscillation in the alpha spectrum during hyperpolarization, indicates membrane potential when these cells are less receptive [40]. Therefore, this type of fluctuation in primary visual areas may be a mechanism to stop incoming information. As a result, the provision of alpha waves cannot stimulate the brain and increase working memory function.

On the other hand, the synchronization of brain 
waves in the theta band did not lead to a significant increase in visuospatial working memory in this study. The activity of the theta band is related to the overall task of the requested working memory, rather than to the encoding individual items. On the other hand, the Corsi blocks tapping test requires memorizing the sequence of different items. Therefore, the brainwave synchronization in the theta band was unable to have a significant increase in the capacity of visuospatial working memory. One of the limitations of the present study was the impossibility of recording EEG to investigate changes in brain waves in subjects.

\section{Conclusions}

The results of this study showed that brainwave synchronization could be used as a non-invasive method to improve cognitive functions including working memory. According to these findings, synchronization of brain waves in the beta band was more effective than alpha and theta bands in improving working memory.

\section{Compliance with ethical guidelines}

All ethical principles were considered in this study. First, the stages of evaluation, intervention, and purpose of the research were explained to the subjects and they completed and signed the informed consent form.

This article was extracted from the research project implemented under contract number 97/85-2 from the special research credit of Azerbaijan Shahid Madani University.

\section{References}

1. Beauchene C, Abaid N, Moran R, Diana RA, Leonessa A. The effect of binaural beats on verbal working memory and cortical connectivity. Journal of Neural Engineering. 2017; 14(2):026014. [DOI:10.1088/1741-2552/aa5d67] [PMID]

2. Baddeley A. Working memory: theories, models, and controversies. Annual Review of Psychology. 2012; 63:1-29. [DOI:10.1146/annurev-psych-120710-100422] [PMID]

3. Vogel EK, McCollough AW, Machizawa MG. Neural measures reveal individual differences in controlling access to working memory. Nature. 2005; 438(7067):500-3. [DOI:10.1038/nature04171] [PMID]

4. Curtis CE, D'Esposito M. Persistent activity in the prefrontal cortex during working memory. Trends in Cognitive Sciences. 2003; 7(9):415-23. [DOI:10.1016/s1364-
[Do 6613(03)00197-9] [PMID]

5. Honey G, Fu C, Kim J, Brammer M, Croudace T, Suckling J, et al. Effects of verbal working memory load on corticocortical connectivity modeled by path analysis of functional magnetic resonance imaging data. Neuroimage. 2002; 17(2):573-82. [PMID]

6. Kuramoto Y. Chaos and statistical methods: proceedings of the sixth Kyoto Summer Institute, Kyoto, Japan September 12-15, 1983. Berlin, Germany: Springer Science \& Business Media; 2012

7. Engel AK, Roelfsema PR, Fries P, Brecht M, Singer W. Role of the temporal domain for response selection and perceptual binding. Cereb Cortex. 1997; 7(6):571-82. [DOI:10.1093/cercor/7.6.571] [PMID]

8. Perfetti B, Moisello C, Landsness EC, Kvint S, Lanzafame S, Onofrj $\mathrm{M}$, et al. Modulation of gamma and theta spectral amplitude and phase synchronization is associated with the development of visuo-motor learning. The Journal of Neuroscience. 2011; 31(41):14810-9. [DOI:10.1523/ JNEUROSCI.1319-11.2011] [PMID] [PMCID]

9. Costa T, Rognoni E, Galati D. EEG phase synchronization during emotional response to positive and negative film stimuli. Neuroscience Letters. 2006; 406(3):159-64. [DOI:10.1016/j.neulet.2006.06.039] [PMID]

10. Fell J, Axmacher N. The role of phase synchronization in memory processes. Nature Reviews Neuroscience. 2011; 12(2):105-18. [DOI:10.1038/nrn2979] [PMID]

11. Kunig $P$, Engel AK, Singer W. Integrator or coincidence detector? The role of the cortical neuron revisited. Trends in Neurosciences. 1996; 19(4):130-7. [DOI:10.1016/s01662236(96)80019-1] [PMID]

12. Addante RJ, Watrous AJ, Yonelinas AP, Ekstrom AD, Ranganath C. Prestimulus theta activity predicts correct source memory retrieval. Proceedings of the National Academy of Sciences of the United States of America. 2011; 108(26): 10702-7. [DOI:10.1073/pnas.1014528108] [PMID] [PMCID]

13. Sauseng P, Klimesch W, Doppelmayr M, Hanslmayr S, Schabus M, Gruber WR. Theta coupling in the human electroencephalogram during a working memory task. Neuroscience Letters. 2004; 354(2):123-6. [DOI:10.1016/j.neulet.2003.10.002] [PMID]

14. Lutzenberger W, Ripper B, Busse L, Birbaumer N, Kaiser J. Dynamics of gamma-band activity during an audiospatial working memory task in humans. The Journal of Neuroscience. 2002; 22(13):5630-8. [DOI:10.1523/JNEUROSCI.22-1305630.2002] [PMID] [PMCID]

15. Oster G. Auditory beats in the brain. Scientific American. 1973; 229(4):94-103. [DOI:10.1038/scientificamerican1073-94] [PMID]

16. Borisyuk A, Semple MN, Rinzel J. Adaptation and inhibition underlie responses to time-varying interaural phase cues in a model of inferior colliculus neurons. Journal of Neurophysiology. 2002; 88(4):2134-46. [DOI:10.1152/jn.2002.88.4.2134] [PMID]

17. Spitzer MW, Semple MN. Transformation of binaural response properties in the ascending auditory pathway: influence of timevarying interaural phase disparity. Journal of Neurophysiology. 1998; 80(6):3062-76. [DOl:10.1152/jn.1998.80.6.3062] [PMID]

18. Fitzpatrick DC, Roberts JM, Kuwada S, Kim DO, Filipovic B. Processing temporal modulations in binaural and monaural auditory stimuli by neurons in the inferior colliculus and auditory cortex. Journal of the Association for Research in Otolaryngology. 2009; 10(4):579-93. [DOI:10.1007/s10162009-0177-8] [PMID] [PMCID]

19. Frederick JA, Lubar JF, Rasey HW, Brim SA, Blackburn J. Effects of $18.5 \mathrm{~Hz}$ auditory and visual stimulation on EEC amplitude at the vertex. Journal of Neurotherapy. 1999; 3(34):23-8. [DOI:10.1300/J184v03n03_03]

20. Becher AK, Huhne M, Axmacher N, Chaieb L, Elger CE, Fell J. Intracranial electroencephalography power and phase synchronization changes during monaural and binaural beat stimulation. The European Journal of Neuroscience. 2015; 41(2):254-63. [DOI:10.1111/ejn.12760] [PMID]

21. Chaieb L, Wilpert EC, Reber TP, Fell J. Auditory beat stimulation and its effects on cognition and mood states. Frontiers in Psychiatry. 2015; 6:70. [DOI:
[D 10.3389/fpsyt.2015.00070] [PMID] [PMCID]

22. Beauchene C, Abaid N, Moran R, Diana RA, Leonessa A. The effect of binaural beats on visuospatial working memory and cortical connectivity. PloS One. 2016; 11(11): e0166630. [DOI:10.1371/journal.pone.0166630] [PMID] [PMCID]

23. Klimesch W, Sauseng P, Hanslmayr S. EEG alpha oscillations: the inhibition-timing hypothesis. Brain Research Reviews. 2007; 53(1):63-88. [DOl:10.1016/j.brainresrev.2006.06.003] [PMID]

24. Beauchene C, Abaid N, Moran R, Diana RA, Leonessa A. The effect of binaural beats on verbal working memory and cortical connectivity. Journal of Neural Engineering. 2017; 14(2):026014. [DOI:10.1088/1741-2552/aa5d67] [PMID]

25. Carter JL, Russell HL. A pilot investigation of auditory and visual entrainment of brain wave activity in learning disabled boys. Texas Researcher. 1993; 4(1):65-75.

26. McMurray JC, Jetha SS, Katz GS, Kemtes KA. Improving memory and attention on two standardized tasks with auditory binaural beats. Archives of Clinical Neuropsychology. 2006; 21(6):516.

27. Dai Z, De Souza J, Lim J, Ho PM, Chen Y, Li J, et al. EEG 
cortical connectivity analysis of working memory reveals topological reorganization in theta and alpha bands. Frontiers in Human Neuroscience. 2017; 11:237. [DOI:10.3389/fnhum.2017.00237] [PMID] [PMCID]

28. Corsi P. Memory and the medial temporal region of the brain. [Doctoral Dissertation]. Montreal, QB: McGill University; 1972.

29. Toepper M, Gebhardt H, Beblo T, Thomas C, Driessen $M$, Bischoff $M$, et al. Functional correlates of distractor suppression during spatial working memory encoding. Neuroscience. 2010; 165(4):1244-53. [DOI:10.1016/j.neuroscience. 2009.11.019] [PMID]

30. Vandierendonck A, Kemps E, Fastame MC, Szmalec A. Working memory components of the Corsi blocks task. British Journal of Psychology. 2004; 95(1):57-79. [DOI:10.1348/000712604322779460] [PMID]

31. Kessels RP, Van Zandvoort MJ, Postma A, Kappelle LJ, De Haan EH. The Corsi block-tapping task: standardization and normative data. Applied Neuropsychology. 2000; 7(4):2528. [DOI:10.1207/S15324826AN0704_8] [PMID]

32. Walker SP, Chang SM, Younger N, Grantham- Mcgregor SM. The effect of psychosocial stimulation on cognition and behaviour at 6 years in a cohort of term, low- birthweight Jamaican children. Developmental Medicine and Child Neurology. 2010; 52(7):e148-54. [DOI:10.1111/j.14698749.2010.03637.x] [PMID]

33. Kraus J, Porubanov6 M. The effect of binaural beats on working memory capacity. Studia Psychologica. 2015; 57(2):135. [DOI:10.21909/sp.2015.02.689]

34. Jirakittayakorn $\mathrm{N}$, Wongsawat $\mathrm{Y}$. Brain responses to a $6-\mathrm{Hz}$ binaural beat: effects on general theta rhythm and frontal midline theta activity. Frontiers in Neuroscience. 2017; 11:365. [DOI:10.3389/fnins.2017.00365] [PMID] [PMCID] 35. Dadashi SD, Ahmadi E, Bafandeh Gharamaleki H, Rasouli $\mathrm{H}$. Effectiveness of brainwave synchronization in beta band by binaural beats on improvement of visuospatial working memory in subjects with borderline personality disorder traits. Studies in Medical Sciences. 2018; 29(5):327-35.

36. Lane JD, Kasian SJ, Owens JE, Marsh GR. Binaural auditory beats affect vigilance performance and mood. Physiology \& Behavior. 1998; 63(2):249-52. [DOI:10.1016/s0031. 9384(97)00436-8] [PMID]

37. Goldman-Rakic PS. Regional and cellular fractionation of working memory. Proceedings of the National Academy of Sciences of the United States of America. 1996; 93(24):13473-80. [DOI:10.1073/pnas.93.24.13473] [PMID] [PMCID]

38. Siegel M, Warden MR, Miller EK. Phase-dependent neuronal coding of objects in short-term memory. Proceedings of the National Academy of Sciences of the United States of America. 2009; 106(50):21341-6. [DOI: 10.1073/pnas.0908193106] [PMID] [PMCID]

39. Lopes da Silva FH, Vos J, Mooibroek J, Rotterdam AV. Relative contributions of intracortical and thalamo-cortical processes in the generation of alpha rhythms, revealed by partial coherence analysis. Electroencephalography and Clinical Neurophysiology. 1980; 50(5-6):449-56. [DOI:10.1016/0013-4694(80)90011-5] [PMID]

40. da Silva FL. Neural mechanisms underlying brain waves: from neural membranes to networks. Electroencephalography and Clinical Neurophysiology. 1991; 79(2):81-93. [DOI:10.1016/0013-4694(91)90044-5] [PMID] 